\title{
Fatores de proteção associados a problemas emocionais e comportamentais em escolares
}

\author{
Protective factors associated with emotional and \\ behavioral problems in school children
}

\author{
Maria Cristina Triguero Veloz TEIXEIRA \\ Mayra Fernanda Ferreira SERACENI ${ }^{1}$ \\ Raquel SURIANO² \\ Nathália Zoli SANT'ANA ${ }^{1}$ \\ Luiz Renato Rodrigues CARREIRO' \\ Cristiane Silvestre de PAULA ${ }^{1}$
}

\begin{abstract}
Resumo
O estudo teve como objetivo verificar associações entre fatores de risco e proteção e problemas emocionais/ comportamentais em escolares. A amostra foi composta por 181 alunos do $2^{\circ}$ a $5^{\circ}$ anos (6 a 12 anos de idade) e seus respectivos cuidadores. Os instrumentos de coleta de dados foram o Inventário de Comportamentos para Crianças e Adolescentes de 6 a 18 anos, um questionário de avaliação socioeconômico familiar, de médias finais de Matermática e Português fornecidas pela escola. Foram investigados problemas emocionais/comportamentais e competências sociais com base no inventário, no desempenho acadêmico e na classificação socioeconômica familiar. Foi conduzida uma análise de regressão logística para gerar um modelo que buscasse associações entre o desfecho (problemas emocionais e comportamentais) e idade, sexo, classificação socioeconômica familiar, desempenho acadêmico e indicadores de socialização. Os resultados indicaram associações entre melhor desempenho acadêmico e indicadores positivos de socialização, com menor probabilidade de as crianças apresentarem problemas emocionais/comportamentais.
\end{abstract}

Palavras-chave: Ajuste emocional; Comportamento infantil; Terapia comportamental; Fatores de risco.

\begin{abstract}
The objective of the study was to verify associations between protective and risk factors with emotional/behavioral problems in schoolchildren. The sample was comprised of 181 students from the $2^{\text {nd }}$ to $5^{\text {th }}$ grades (aged 6-12) and their caregivers. The instruments used were the Child Behavior Checklist for ages 6-18, a socioeconomic questionnaire, besides the average final grades on Mathematics and Portuguese provided by the school. We assessed emotionall
\end{abstract}

\footnotetext{
$\boldsymbol{v} \mathbf{v} \boldsymbol{v}$
}

1 Universidade Presbiteriana Mackenzie, Centro de Ciências Biológicas e da Saúde, Programa de Pós-Graduação em Distúrbios do Desenvolvimento. R. da Consolação, 896, Prédio 28, Consolação, 01302-907, São Paulo, SP, Brasil. Correspondência para/Correspondence to: M.C.T.V. TEIXEIRA. E-mail: <mctvteixeira@gmail.com>.

2 Universidade Presbiteriana Mackenzie, Centro de Ciências Biológicas e da Saúde, Curso de Psicologia. São Paulo, SP, Brasil.

Artigo elaborado a partir da dissertação de N.Z. SANT'ANA, intitulada "Estudo transversal sobre problemas de comportamento, desempenho acadêmico e competências sociais em escolares do Ensino Fundamental I". Universidade Presbiteriana Mackenzie, 2012. 
behavioral problems and social competence from the Child Behavior Checklist for ages 6-18, academic performance, and family socioeconomic status. We conducted a multivariate logistic regression analysis to generate a model seeking associations between the outcome (emotional and behavioral problems) and age, gender, family socioeconomic status, academic performance and socialization indicators. Results indicated better academic performance and positive socialization indicators associated with a lower probability of emotional and behavioral problems in this sample.

Keywords: Emotional adjustment; Child behavior; Risk factors; Behavior therapy.

A prevenção de problemas emocionais e comportamentais em crianças e adolescentes deve tornar-se prioridade nas políticas públicas brasileiras de saúde e educação, haja vista as evidências que apontam taxas elevadas de prevalência desses problemas (Assis \& Constantino, 2005; Bolsoni-Silva, 2003; Bolsoni-Silva, Silveira, \& Marturano, 2008; Paula, Duarte, \& Bordin, 2007; Paula, Miranda, \& Bordin, 2010; Rios \& Williams, 2008).

Constata-se no Brasil um aumento progressivo do interesse por estudos voltados à identificação, prevenção e intervenção em problemas emocionais e comportamentais na infância (Barreto, Freitas, \& Del Prette, 2011; Costa, Williams, \& Cia, 2012; Couto, Duarte, \& Delgado, 2008; Murta, 2007; Paula et al., 2010; Razzouk, Zorzetto, Dubugras, Gerolin, \& Mari, 2006; Rios \& Williams, 2008). Problemas de comportamento na infância representam déficits ou excessos comportamentais que prejudicam não só a interação da criança com seus pares e adultos, como também a aquisição de repertórios adequados de aprendizagem. Tais problemas, quando não identificados, costumam provocar prejuízos funcionais em diversas áreas da vida da criança e do adolescente (Bolsoni-Silva, 2003).

Estudos sobre prevenção de problemas de comportamento na infância têm se voltado para a avaliação de fatores de risco e de proteção associados a esses problemas. Fator de risco deve ser compreendido como um conceito relacional, na medida em que se refere a um evento que pode aumentar a probabilidade de um determinado desfecho inadequado, quando comparado à mesma população não exposta ao evento (Sá, Bordin, Martin, \& Paula, 2010). O status de fator de risco de um determinado evento é condicional, pois depende de outros eventos com os quais ele se relaciona, podendo, inclusive, modificar o curso do desfecho.

540 Daí a existência, numa mesma população, de grupos vulneráveis e não vulneráveis (Sá et al., 2010). Por isso, o termo fator de risco tem sido utilizado principalmente para referir a condições antecedentes associadas ao aumento da chance de ocorrência de resultados indesejáveis ou efeitos adversos (Kazdin, Kraemer, Kessler, Kupfer, \& Offord, 1997; Kraemer et al., 1997). Já os fatores de proteção são eventos que podem atenuar o impacto negativo dos fatores de risco (Kim-Cohen, 2007).

Alguns dos fatores de risco mais estudados, relacionados a problemas de saúde mental e de comportamento na infância, são as habilidades sociais deficitárias, as dificuldades no desempenho acadêmico, as práticas educativas parentais inadequadas, dentre outros (Bolsoni-Silva, Loureiro, \& Marturano, 2011; Brancalhone, Fogo, \& Williams, 2004; Cecconello, De Antoni, \& Koller, 2003; Cia, Pereira, Z.A.P. Del Prette, \& Del Prette, 2006; Z.A.P. Del Prette \& Del Prette, 2003; Ferriolli, 2006; Marturano \& Loureiro, 2003; Milán, García, Hurtado, Morilla, \& Sepúlveda, 2006; O'Connel, Boat, \& Warner, 2009). Como fatores de proteção têm sido estudados o suporte social e a participação em atividades pró-sociais (atividades de lazer, prática de esporte, brincadeiras ao ar livre, leituras e práticas educativas adequadas) (Cobb, 1976; Cohen \& Wills, 1985; Jackson \& Warren, 2000; Kazdin et al., 1997; Kim-Cohen, 2007; Paula et al., 2008; Sá et al., 2010).

Segundo O'Connel et al. (2009), inúmeros fatores, como competências individuais, condições financeiras, qualidade da educação, entre outros, podem aumentar ou diminuir o risco de a criança ou o adolescente desenvolver problemas de saúde mental ou de comportamento, tais como uso de substâncias, violência, desafio, oposição e agressividade. Para os autores, esses fatores tendem a ter um efeito cumulativo: a criança que é exposta a um maior número de fatores de risco tem maiores 
possibilidades de sofrer interferências que prejudiquem seu desenvolvimento. Por outro lado, a exposição da criança a um número maior de fatores de proteção, como práticas parentais positivas, por exemplo, poderá diminuir as probabilidades de um desfecho prejudicial em termos de padrões comportamentais inadequados.

Quando os pais estabelecem um ambiente familiar adequado (clima emocional familiar positivo, suporte à autonomia, estruturação de regras e rotinas no lar) e propiciam momentos favoráveis ao desenvolvimento infantil, eles estão criando fatores de proteção frente a situações ameaçadoras às quais as crianças podem ser expostas (Z.A.P. Del Prette \& Del Prette, 2003; Marturano \& Loureiro, 2003).

Tanto no Brasil como em outros países, trabalhos de revisão sobre estudos epidemiológicos no tema da saúde mental na infância e adolescência apontam que é necessária a realização de estudos que identifiquem fatores de risco e proteção para problemas de saúde mental nessa faixa etária (Paula et al., 2010; Weare \& Nind, 2011). A identificação desses fatores na infância pode possibilitar o desenvolvimento de programas e ações de intervenção destinadas à prevenção e à amenização de problemas e dificuldades emocionais e comportamentais que, quando não tratados adequadamente, poderão evoluir para transtornos psiquiátricos (Kim-Cohen, 2007; O'Connel et al., 2009).

O presente estudo teve como objetivo verificar a associação entre fatores de risco e proteção para problemas emocionais e comportamentais em um grupo de escolares do Ensino Fundamental I.

\section{Método}

\section{Participantes}

Este é um estudo do tipo seccional ou transversal (Klein \& Bloch, 2006), cuja amostra foi composta por 181 escolares e seus pais/cuidadores. As crianças estavam matriculadas entre $\circ 2^{\circ}$ e $05^{\circ}$ ano do Ensino Fundamental I de uma escola particular da cidade de São Paulo, na faixa etária entre 6 e 12 anos de idade (média: 8,53; desvio-padrão: 1,23). Do total de pais que responderam aos instrumentos, 96,1\% foram compostos por mãe ou pai biológico. A faixa etária dos respondentes concentrou-se entre 35-45 anos (59,7\%) seguida pela faixa etária de 24 a 34 anos (21,5\%). Em relação ao nível de escolaridade dos respondentes, 97,7\% concentraram-se entre os níveis médio e superior. A coleta de dados foi conduzida entre os meses de agosto e outubro de 2011. O projeto foi aprovado pelo Comitê de Ética em Pesquisa com Seres Humanos da Universidade Presbiteriana Mackenzie (Processo CEP/UPM 1374/08/2011 e CAAE 0069.0.272.000-11).

\section{Instrumentos}

O trabalho contou com três instrumentos:

a) Inventário de Comportamentos para Crianças e Adolescentes entre 6 e 18 anos (CBCL/6-18): esse instrumento avalia indicadores comportamentais de competências nas áreas social, escolar e de realização de atividades, assim como problemas emocionais e comportamentais da criança ou adolescente, referente aos últimos seis meses (Achenbach \& Rescorla, 2001). O inventário foi respondido por um cuidador responsável pela criança. As escalas de competências do inventário são compostas por oito perguntas que avaliam realização de atividades (número e tipo de esportes, brincadeiras, passatempos, número de organizações e/ou clubes frequentados e tarefas da vida cotidiana realizadas pela criança ou adolescente), indicadores de socialização (número de amigos, número de vezes que se encontra com estes fora da escola, qualidade das interações sociais com pares, irmãos e pais) e indicadores de desempenho escolar (as habilidades nas matérias escolares, de acordo com o relato dos cuidadores, são avaliadas como melhor, igual, pior ou abaixo da média exigida pela escola). Os problemas de comportamento abrangem 112 perguntas agrupadas em cinco escalas: escalas das síndromes, escalas orientadas pelo Diagnostic and Statistical Manual of Mental Disorders (DSM), escalas de problemas de comportamento interna- 
lizantes, escala de problemas de comportamento externalizantes e escala total de problemas emocionais e comportamentais. Alguns dos problemas de comportamento avaliados nessas escalas são ansiedade e depressão, queixas somáticas de fundo emocional, problemas de sociabilidade, problemas de atenção, isolamento e depressão, violação de regras, desafio e oposição, problemas afetivos, problemas de déficit de atenção e hiperatividade, dentre outros. A instrução do inventário para as escalas de problemas de comportamento estabelece que os itens sejam preenchidos atribuindo 0 , se o item não é verdadeiro para a criança ou adolescente; 1 , se é um pouco verdadeiro ou às vezes verdadeiro; 2 , se é muito verdadeiro ou frequentemente verdadeiro. A versão brasileira oficial do CBCL/6-18 foi concluída em 2011, passando por um complexo processo de tradução, retrotradução e adaptação cultural, sob a liderança de pesquisadores brasileiros, em parceria com os autores da versão original do instrumento (Bordin et al., 2013). Como replicado em estudo brasileiro anterior (Santos \& Silvares, 2006), definiram-se como casos positivos aqueles que obtiveram pontuação na faixa limítrofe e clínica da escala total de problemas emocionais e comportamentais do CBCL/6-18.

b) Registro de Desempenho Acadêmico: foi identificada a média final de notas das disciplinas de Matemática e Português. Esses dados foram disponibilizados pela coordenação da escola.

c) Questionário de Classificação Econômica Familiar: questionário desenvolvido pela Associação Brasileira de Estudos Populacionais (2009) para a determinação da classe econômica, segundo o poder de consumo da família. O questionário inclui uma combinação de itens para identificar a classe social da família: escolaridade do chefe da família, poder econômico familiar para adquirir bens de consumo (eletrodomésticos, veículos), número de banheiros no domicílio. Cinco classes sociais podem ser estabelecidas segundo o escore total obtido: A (35-46), B (23-34), C (14-22), D (8-13) e E (0-7). Quanto mais baixo o escore, menor o poder de compra

542 da família.

\section{Procedimentos}

Em reunião previamente agendada pela coordenação da escola, foi realizada a apresentação e convite aos pais para participarem do estudo. Compareceram 235 pais, dos quais 216 concordaram em participar do estudo. Foram devolvidos 185 inventários, quatro dos quais tiveram de ser excluídos em decorrência de preenchimento inadequado dos dados. Após o consentimento dos pais/cuidadores, foram explicados todos os procedimentos para o preenchimento do instrumento de coleta de dados, conforme o respectivo fundamento teórico-metodológico. Os cuidadores informantes foram instruídos sobre o preenchimento do inventário CBCL/6-18, tanto para não deixar itens em branco, quanto para não aceitar interferência de outro cuidador que desejasse complementar a resposta. As instruções fornecidas tiveram como finalidade reduzir o viés na coleta de dados (Rocha, Pereira, Arantes, \& Silvares, 2010). Todos os inventários foram encaminhados em envelope fechado, junto à agenda dos alunos. Uma vez devolvidos os instrumentos respondidos, procedeu-se à revisão e identificação de itens não preenchidos. Foram realizadas aproximadamente 80 ligações telefônicas para completar os dados com os mesmos cuidadores responsáveis que haviam preenchido o instrumento.

Todos os dados coletados foram organizados em um banco, com o auxílio do programa Statistical Package for the Social Sciences (SPSS), versão 19.0. Inicialmente foram conduzidas análises descritivas dos dados, mediante o uso de tabelas de frequências simples e comparação de distribuição de sexo e faixa etária a partir do teste de Igualdade de Duas Proporções. Para verificar a distribuição de crianças e adolescentes com problemas emocionais e comportamentais (com pontuação clínica e/ou limítrofe na escala total de problemas emocionais e comportamentais do CBCL/6-18), foi utilizado o software Assessment Data Manager (ADM), versão 7.2. Esse software permite a conversão dos escores brutos das escalas em escores $T$ (escores padronizados por idade e sexo) e posteriormente classifica as escalas em normal, limítrofe e clínica (Achenbach \& Rescorla, 2001). 
Após as análises descritivas, foi realizada uma análise multivariada (regressão logística) com a finalidade de gerar um modelo buscando associações entre o desfecho problemas emocionais e comportamentais e os seguintes fatores: idade, sexo, classificação socioeconômica familiar, desempenho acadêmico e indicadores de socialização segundo escala social de competências do CBCL/6-18 (os itens do CBCL/6-18 que permitem verificar uma socialização adequada ou não são o número de amigos da criança/adolescente, o número de vezes que ela se encontra com esses amigos fora da escola e a qualidade das interações sociais com pares, irmãos e pais).

\section{Resultados}

Na Tabela 1 apresenta-se uma distribuição da frequência relativa de classificação do grupo nas diferentes escalas do CBCL/6-18, lembrando que a classificação limítrofe foi agrupada na classificação clínica, como descrito no método.

De acordo com a Tabela 1, os problemas de comportamento mais frequentes verificados na amostra do estudo foram: a) 69 crianças com problemas de comportamento internalizantes $(38,1 \%)$; b) 68 crianças com déficit de competências para realização de atividades (37,6\%); c) 51 crianças com problemas de ansiedade e depressão $(28,2 \%)$; d) 47 crianças com problemas de ansiedade $(26,0 \%)$; e) 47 crianças com problemas totais emocionais e comportamentais $(26,0 \%)$.

Na Tabela 2 observam-se os potenciais fatores de risco e proteção para problemas emocionais e comportamentais identificados na amostra, que serviram de base para gerar o modelo inicial de regressão logística.

Tabela 1

Distribuição da frequência relativa de classificação nas faixas limítrofe/clínica e normal no grupo, de acordo com as escalas do CBCL/6-18

\begin{tabular}{|c|c|c|c|c|}
\hline \multirow{2}{*}{ Escalas do CBCL/6-18 } & \multicolumn{2}{|c|}{ Normal } & \multicolumn{2}{|c|}{ Limítrofe/Clínico } \\
\hline & $\mathrm{N}$ & $\%$ & $\mathrm{~N}$ & $\%$ \\
\hline \multicolumn{5}{|l|}{ Escalas de competências } \\
\hline Realização de atividades & 113 & 62,4 & 68 & 37,6 \\
\hline Social & 153 & 84,5 & 28 & 15,5 \\
\hline Desempenho escolar & 151 & 83,4 & 29 & 16,0 \\
\hline \multicolumn{5}{|l|}{ Escalas das síndromes } \\
\hline Ansiedade e depressão & 130 & 71,8 & 51 & 28,2 \\
\hline Isolamento e depressão & 164 & 90,6 & 17 & 9,4 \\
\hline Queixas somáticas & 157 & 86,7 & 24 & 13,3 \\
\hline Problemas de sociabilidade & 166 & 91,7 & 15 & 8,3 \\
\hline Problemas com pensamento & 157 & 86,7 & 24 & 13,3 \\
\hline Problemas de atenção & 159 & 87,8 & 22 & 12,2 \\
\hline Violação de regras & 175 & 96,7 & 6 & 3,3 \\
\hline Comportamento agressivo & 161 & 89,0 & 20 & 11,0 \\
\hline \multicolumn{5}{|l|}{ Escalas orientadas pelo DSM } \\
\hline Problemas afetivos & 153 & 84,5 & 28 & 15,5 \\
\hline Problemas de ansiedade & 134 & 74,0 & 47 & 26,0 \\
\hline Problemas somáticos & 157 & 86,7 & 24 & 13,3 \\
\hline Problemas de déficit de atenção e hiperatividade & 158 & 87,3 & 23 & 12,7 \\
\hline Problemas oposição e desafio & 170 & 93,9 & 11 & 6,1 \\
\hline Problemas de conduta & 173 & 95,6 & 8 & 4,4 \\
\hline Internalização & 112 & 61,9 & 69 & 38,1 \\
\hline Externalização & 153 & 84,5 & 28 & 15,5 \\
\hline Total de problemas emocionais/comportamentais & 134 & 74,0 & 47 & 26,0 \\
\hline
\end{tabular}

Nota: CBCL/6-18: Inventário de Comportamentos para crianças e Adolescentes entre 6 e 18 anos; DSM: Diagnostic and Statistical Manual of Mental Disorders. 
O conjunto de variáveis independentes descritos na Tabela 2 foi utilizado como base para o modelo inicial de regressão logística. Sob a con-

\section{Tabela 2}

Descrição dos potenciais fatores de risco e proteção para problemas emocionais e comportamentais da amostra de crianças participantes $(N=181)$

\begin{tabular}{|c|c|c|}
\hline \multirow{2}{*}{ Características crianças/adolescentes } & \multicolumn{2}{|c|}{ Amostra } \\
\hline & $N$ & $\%$ \\
\hline \multicolumn{3}{|l|}{ Sexo } \\
\hline Feminino & 86 & 47,5 \\
\hline Masculino & 95 & 52,5 \\
\hline \multicolumn{3}{|l|}{ Idade (anos) } \\
\hline 06-09 & 140 & 77,3 \\
\hline $10-12$ & 41 & 22,7 \\
\hline \multicolumn{3}{|l|}{ Classificação socioeconômica da família } \\
\hline A & 73 & 40,3 \\
\hline B & 91 & 50,3 \\
\hline C & 17 & 9,4 \\
\hline \multicolumn{3}{|c|}{$\begin{array}{l}\text { Total de problemas emocionais/comportamentais/ } \\
\text { CBCL/6-18 }\end{array}$} \\
\hline Normal & 134 & 74,0 \\
\hline Limítrofe/clínico & 47 & 26,0 \\
\hline \multicolumn{3}{|l|}{ Escala de competências social//CBCL/6-18 } \\
\hline Normal (adequada) & 153 & 84,5 \\
\hline Limítrofe/clínico & 28 & 15,5 \\
\hline \multicolumn{3}{|l|}{ Média de nota em matemática } \\
\hline Na média ( $\geq 6,0$ pontos) & 166 & 91,7 \\
\hline Abaixo da média & 15 & 8,3 \\
\hline \multicolumn{3}{|l|}{ Média de nota em português } \\
\hline Na média ( $\geq 6,0$ pontos) & 170 & 93,9 \\
\hline Abaixo da média & 11 & 6,1 \\
\hline
\end{tabular}

Nota: CBCL/6-18: Inventário de Comportamentos para crianças e Adolescentes entre 6 e 18 anos. dição de fatores de risco ou proteção, observam-se na Tabela 3 as razões de chances dessas variáveis em relação aos problemas emocionais e comportamentais.

De acordo com a Tabela 4, no modelo final da regressão logística, permaneceram somente duas variáveis como potenciais fatores protetores associados aos problemas emocionais e comportamentais totais dos indivíduos da amostra: desempenho acadêmico em Matemática (medido pela nota média final nessa matéria) e competências de socialização (medida na escala social de competências do CBCL/6-18, a partir da percepção dos pais sobre número de amigos, número de vezes que se encontra com eles fora da escola e qualidade das interações sociais com pares, irmãos e pais).

\section{Discussão}

Os resultados do presente estudo são semelhantes aos obtidos em outros trabalhos brasileiros que indicam associações entre fatores protetores, como desempenho acadêmico e socialização, em relação a problemas emocionais e comportamentais (Bandeira, Rocha, Souza, Z.A.P. Del Prette, \& Del Prette, 2006; D'Avila-Bacarji; Marturano, \& Elias, 2005; Elias \& Marturano, 2005; Ferreira \& Marturano, 2002; Gardinal \& Marturano, 2007; Medeiros, Loureiro, Linhares, \& Marturano 2000; Santos \& Graminha, 2006).

Sendo o presente estudo do tipo transversal, não é possível afirmar que melhor desempenho acadêmico e socialização adequada sejam fatores

\section{Tabela 3}

Modelo de regressão logística inicial com Odds Ratios e Intervalo de Confiança de 95\% (IC95\%) para descrição da associação entre os fatores de risco/proteção em relação aos problemas emocionais e comportamentais $(N=181)$

\begin{tabular}{|c|c|c|c|c|c|}
\hline & Variáveis & $\beta$ & Valor de $p$ & Razão de chances & IC95\% \\
\hline \multirow{6}{*}{ Passo 1 (a) } & Idade da criança & $-0,302$ & 0,470 & 0,740 & $0,55-0,996$ \\
\hline & Classificação Socioeconômica da Família & 0,259 & 0,360 & 1,296 & $0,74-2,26$ \\
\hline & Sexo da criança & 0,074 & 0,845 & 1,077 & $0,51-2,28$ \\
\hline & Escala Competência Social (CBCL/6-18) & $-1,181$ & 0,009 & 0,307 & $0,13-0,75$ \\
\hline & Desempenho português nota média final & 0,256 & 0,361 & 1,292 & $0,75-2,24$ \\
\hline & Desempenho matemática nota média final & $-0,471$ & 0,056 & 0,625 & $0,39-1,01$ \\
\hline
\end{tabular}

Nota: *Valor de p estatisticamente significante com 95\% de confiança estatística.

544

CBCL/6-18: Inventário de Comportamentos para Crianças e Adolescentes entre 6 e 18 anos. 
Tabela 4

Modelo de regressão logística final com Odds Ratios e Intervalo de Confiança de 95\% (IC95\%) identificando o melhor modelo associativo entre os fatores de risco/proteção em relação aos problemas emocionais e comportamentais $(N=181)$

\begin{tabular}{llccc}
\hline & Variáveis & $\beta$ & Valor de $p$ & Razão de chances \\
\hline \multirow{2}{*}{ Passo 1 (a) } & Escala Competência Social (CBCL/6-18) & $-1,289$ & 0,003 & 0,275 \\
\cline { 2 - 4 } & Desempenho matemática nota média final & $-0,265$ & 0,040 & $0,12-0,64$ \\
\hline
\end{tabular}

Nota: *Valor de $p$ estatisticamente significante com 95\% de confiança estatística.

CBCL/6-18: Inventário de Comportamentos para Crianças e Adolescentes entre 6 e 18 anos.

protetores para o desenvolvimento de problemas emocionais/comportamentais entre as crianças e adolescentes participantes. Entretanto, a literatura tem indicado que a associação entre esses três fatores se dá nessa direção, já que desempenhos escolares adequados estão associados a uma melhor adaptação escolar, o que parece contribuir para a minimização de diversos problemas comportamentais. É provável que, por essa razão, desde a década de 1990, existam evidências de que a qualidade do desempenho acadêmico seja um dos fatores preditores do futuro perfil emocional/comportamental de crianças e adolescentes (Cia \& Barham, 2009a, 2009b; Gordon, 1993).

O desempenho acadêmico prejudicado parece ter impacto direto no comportamento de crianças, adolescentes e jovens, devido ao papel central que a escola tem nessa fase da vida. Duas pesquisas brasileiras conduzidas no Rio Grande do Sul ilustram bem esse cenário. Um estudo transversal com expressiva amostra (3 139 crianças e adolescentes de Pelotas, RS) indicou que os adolescentes de 10 a 13 anos com problemas emocionais e comportamentais (segundo o CBCL/6-18) tinham praticamente o dobro de chance de terem repetido na escola, em relação a seus colegas sem esses problemas ( $p<0,01$; Razão de Prevalência $=1,9$; Intervalo de Confiança $=1,5-2,4$ ) (Benvegnú, Fassa, Facchini, Wegman, \& Dall'Agnol, 2005). Paralelamente, o estudo epidemiológico do tipo caso-controle ( $N=93)$ conduzido em Porto Alegre evidenciou maior taxa de evasão escolar na $3^{\mathrm{a}}$ e $4^{\mathrm{a}}$ séries do ensino fundamental de escolas públicas entre estudantes com transtorno de conduta, em comparação com estudantes sem problemas de comportamento: 31,8 e 2,3\% respectivamente (Tramontina et al., 2002).
Dificuldades nas habilidades sociais podem ser compreendidas como déficits na aprendizagem de padrões comportamentais e cognitivos que, se ausentes, conduziriam a uma pior resposta em situações cotidianas que envolvem relacionamentos sociais (Z.A.P. Del Prette \& Del Prette, 2003). Por outro lado, o desenvolvimento de repertórios sociais adequados, que ocorrem primeiramente a partir de relacionamentos pessoais com familiares e com os pares, tende a levar ao maior sucesso nessas mesmas circunstâncias (Pacheco, Teixeira, \& Gomes, 1999). Assim, as habilidades sociais têm forte impacto no desenvolvimento intelectual e psíquico dos indivíduos e, por isso, tendem a reduzir o risco de desenvolvimento de problemas emocionais e comportamentais. Nesse sentido, as interações sociais podem ser consideradas também educativas, na medida em que representam uma aquisição de conceitos, habilidades e estratégias cognitivas que indiretamente afetam a aprendizagem (Z.A.P. Del Prette \& Del Prette, 1998).

De forma geral, a literatura aponta que existe uma inter-relação entre essas áreas do desenvolvimento humano, ou seja, entre habilidades sociais de crianças/jovens e seu desempenho acadêmico. Nota-se que intervenções relativas ao treino de habilidades sociais parecem promover progresso não só na socialização em si, mas também na área acadêmica (Cia \& Barham, 2009b; Molina \& Del Prette, 2006). Por isso, diversos estudos têm sido concluídos visando aprimorar habilidades acadêmicas e sociais. Essa questão é bem exemplificada por um estudo brasileiro com delineamento quase experimental voltado para a intervenção. Na pesquisa, 16 estudantes (7 a 13 anos de idade) com dificuldades de aprendizagem receberam dois tipos de intervenção: uma exclusivamente acadêmica e outra exclusiva- 
mente voltada às habilidades sociais. Ambos os grupos demonstraram progresso após as intervenções; contudo, o primeiro grupo obteve resultados positivos apenas nas habilidades acadêmicas, enquanto o segundo obteve progresso nas habilidades acadêmicas e sociais concomitantemente (Molina \& Del Prette, 2006). Embora tenha sido adotado um desenho metodológico quase experimental, o que limita as possibilidades de controle de variáveis, os resultados indicaram que benefícios no campo da socialização contribuíram com melhora no desempenho acadêmico. A associação entre indicadores de socialização e desempenho acadêmico também foi demonstrada no estudo de Cia, Barham, \& Fontaine (2010). Nesse trabalho foram verificados resultados positivos em habilidades de leitura e em habilidades sociais, após um programa de intervenção com pais de crianças dos anos iniciais do Ensino Fundamental I (Cia et al., 2010).

De forma geral, conclui-se que há certo consenso na literatura em relação à existência de associação entre problemas emocionais/comportamentais e desempenho acadêmico e habilidades sociais, como foi identificado na presente pesquisa. As diretrizes do Sistema Único de Saúde brasileiro preconizam que as políticas públicas devem basear-se em evidências científicas, visando maximizar os recursos humanos e financeiros existentes (Brasil, 2000). Por isso, resultados de pesquisas realizadas com crianças brasileiras são de grande importância para fornecer evidências que contribuam para a implantação de políticas públicas mais efetivas e promissoras aplicáveis a nossa realidade.

Sabe-se que a população infanto-juvenil brasileira encontra-se frequentemente exposta a diversos riscos, tornando imperativa a implementação de programas preventivos, principalmente porque já existem modelos com bons resultados, como aqueles que incluem promoção de habilidades sociais educativas, estímulo à competência social, formação de grupos educativos e de solução de problemas interpessoais no ambiente escolar, entre outros (Murta, 2007). Nesse sentido, a presente pesquisa vai ao encontro de tal premissa, já que permitiu identificar dois fatores protetores em relação a problemas emocionais e comportamentais de estudantes, contribuindo, portanto, para esse campo do conhecimento, apoiando o desenvolvimento de estratégias de prevenção e intervenção nos campos da saúde, educação e psicologia.

\section{Considerações Finais}

Este estudo de corte transversal identificou associações entre fatores protetores e o desfecho clínico de problemas emocionais e comportamentais entre os escolares participantes. Devido à estreita ligação entre os três domínios abordados, intervenções que promovem seja o desempenho acadêmi$\mathrm{co}$, seja as habilidades sociais poderiam reduzir os problemas emocionais e comportamentais dessas crianças.

Diante dos resultados obtidos, sugere-se a implementação de intervenções que estimulem indicadores de socialização na infância, pois, além de constituírem estratégias de prevenção com benefícios importantes para o desenvolvimento da criança, costumam ter baixo custo.

Embora o presente trabalho traga novos dados para o campo de programas preventivos e de intervenção, algumas limitações devem ser apontadas, como: (1) a amostra do estudo é reduzida; (2) os resultados foram baseados em uma amostra de classes A e B predominantemente, não podendo portanto serem extrapolados para outras classes sociais; (3) os dados referentes a problemas comportamentais dos participantes envolvidos dizem respeito a um único informante, e não a múltiplos; (4) os indicadores de socialização utilizados nesta pesquisa (de acordo com o CBCL/6-18) não permitem avaliar outros repertórios de habilidades sociais dos participantes, pois são restritos ao campo das amizades, frequências de contatos sociais e as qualidades das interações com pares, irmãos e pais.

\section{Referências}

Achenbach, T., \& Rescorla, L. (2001). Manual for the ASEBA School: Age forms and profiles. Burlington, V.T.: Research Center for Children, Youth \& Families.

Assis, S. G., \& Constantino, P. (2005). Perspectivas de prevenção da infração juvenil masculina. Ciência e Saúde Coletiva, 10(1), 81-90. 
Associação Brasileira de Estudos Populacionais. (2009). Dados com base no levantamento sócio econômico. Belo Horizonte: Abep; c2012. Recuperado em dezembro 3, 2012, de www.abep.org-Dados-IBOPE

Bandeira, M., Rocha, S. S., Souza, T. M. P., Del Prette, Z. A. P., \& Del Prette, A. (2006). Comportamentos problemáticos em estudantes do ensino fundamental: características da ocorrência e relação com habilidades sociais e dificuldades de aprendizagem. Estudos de Psicologia, 11(2), 199-208.

Barreto, S. O., Freitas, L. C., \& Del Prette, Z. A. P. (2011). Habilidades sociais na comorbidade entre dificuldades de aprendizagem e problemas de comportamento: uma avaliação multimodal. Revista Psico, 42(4), 503-510.

Benvegnú, L. A., Fassa, A. G., Facchini, L. A., Wegman, D. H., \& Dall'Agnol, M. M. (2005). Work and behavioural problems in children and adolescents. International Journal of Epidemiology, 34(6), 1417-1424.

Bolsoni-Silva, A. T. (2003). Habilidades Sociais Educativas, variáveis contextuais e problemas de comportamento: comparando pais e mães de pré-escolares (Tese de doutorado não-publicada). Universidade de São Paulo, Ribeirão Preto.

Bolsoni-Silva, A. T., Silveira, F. F., \& Marturano, E. M. (2008). Promovendo habilidades sociais educativas parentais na prevenção de problemas de comportamento. Revista Brasileira de Terapia Comportamental e Cognitiva, 10(2), 125-142.

Bolsoni-Silva, A. T., Loureiro, S. R., \& Marturano, E. M. (2011). Problemas de comportamento e habilidades sociais infantis: modalidades de relatos. Revista Psico, 42(3), 354-361.

Bordin, I. A., Rocha, M. M., Paula, C. S., Teixeira, M. C. T. V., Achenbach, T. M., Rescorla, L. A., \& Silvares, E. F. M. (2013). Child Behavior Checklist (CBCL), Youth Report (YSR) and Teacher's Report Form (TRF): An overview of the development of the original and Brazilian versions. Cadernos de Saúde Pública, 29(1), 13-28.

Brancalhone, P. G., Fogo, J. C., \& Williams, L. C. A. (2004). Crianças expostas à violência conjugal: avaliação do desempenho acadêmico. Psicologia: Teoria e Pesquisa, 20(2), 113-117.

Brasil. Ministério da Saúde. (2000). Sistema Sistema Único de Saúde (SUS) princípios e conquistas. Brasília: Ministério da Saúde. Recuperado em outubro 13, 2013, de http://bvsms.saude.gov.br/bvs/publicacoes/ sus_principios.pdf

Cecconello, A. M., De Antoni, C., \& Koller, S. H. (2003). Práticas educativas, estilos parentais e abuso físico no contexto familiar. Psicologia em Estudo, 8(Esp.), 45-54.

Cia, F., \& Barham, E. J. (2009a). O envolvimento paterno e o desenvolvimento social de crianças iniciando as atividades escolares. Psicologia em Estudo, 14(1), 67-74.
Cia, F., \& Barham, E. J. (2009b). Repertório de habilidades sociais, problemas de comportamento, autoconceito e desempenho acadêmico de crianças no início da escolarização. Estudos de Psicologia (Campinas), 26(1), 45-55.

Cia, F., Barham, E. J., \& Fontaine, A. M. G. V. (2010). Impactos de uma intervenção com pais: o desempenho acadêmico e comportamento das crianças na escola. Psicologia: Reflexão e Crítica, 23(3), 533-543.

Cia, F., Pereira, C. S., Del Prette, Z. A. P., \& Del Prette, A. (2006). Habilidades sociais parentais e o relacionamento entre pais e filhos. Psicologia em Estudo, 11(1), 73-81.

Cobb, S. (1976) Social Support as a moderator of life stress. Psychosomatic Medicine. 38(5), 300-314.

Cohen, S., \& Wills, T. A. (1985). Stress, social support and the buffering hypothesis. Psychological Bulletin, 98(2), 310-357.

Costa, C. S. L., Williams, L. C. A., \& Cia, F. (2012). Intervenção com monitores de organização não-governamental: diminuindo problemas de comportamento em crianças. Psicologia: Reflexão e Crítica, 25(3), 411-421.

Couto, M. C. V., Duarte, C. S., \& Delgado, P. G. G. (2008). A saúde mental infantil na saúde pública brasileira: situação atual e desafios. Revista Brasileira de Psiquiatria, 30(4), 384-389.

D’Avila-Bacarji, K. M. G., Marturano, E. M., \& Elias, L. C. S. (2005). Suporte parental: um estudo sobre crianças com queixas escolares. Psicologia em Estudo, 10(1), 107-115.

Del Prette, Z. A. P., \& Del Prette, A. (1998). Desenvolvimento interpessoal e educação escolar: o enfoque das habilidades sociais. Temas em Psicologia, 6(3), 205-215.

Del Prette, Z. A. P., \& Del Prette, A. (2003). Desenvolvimento socioemocional na escola e prevenção da violência: questões conceituais e metodologia de intervenção. In A. Del Prette \& Z. A. P. Del Prette (Orgs.), Habilidades sociais, desenvolvimento e aprendizagem: questões conceituais, avaliação e intervenção (pp.83-127). Campinas: Alínea.

Elias, L. C., \& Marturano, E. M. (2005). Oficinas de linguagem: proposta de atendimento psicopedagógico para crianças com queixas escolares. Estudos de Psicologia, 10(1), 53-61.

Ferreira, M. C. T., \& Marturano, E. M. (2002). Ambiente familiar e os problemas de comportamento apresentados por crianças com baixo desempenho escolar. Psicologia: Reflexão e Crítica, 15(1), 35-44.

Ferriolli, S. H. T. (2006). Indicadores de risco e proteção ao desenvolvimento do escolar: crianças e famílias atendidas em um programa de atenção primária e saúde da família (Tese de doutorado não-publicada). Universidade de São Paulo, Ribeirão Preto.

Gardinal, E. C., \& Marturano, E. M. (2007). Meninos e meninas na educação infantil: associação entre compor- 
tamento e desempenho. Psicologia em Estudo, 12(3), 541-551.

Gordon, N. (1993). Learning disorders and delinquency. Brain \& Development, 15(3), 169-172.

Jackson, Y., \& Warren, J. S. (2000). Appraisal, social support, and life events: Predicting outcomes behavior in school-age children. Child Development, 71(5), 1441-1457.

Kazdin, A. E., Kraemer, H. C., Kessler, R. C., Kupfer, D. J., \& Offord, D. R. (1997). Contributions of risk factor research to developmental psychology. Clinical Psychology Review, 17(4) 375-406.

Kim-Cohen, J. (2007). Resilience and developmental psychopathology. Child Adolescent Psychiatric Clinics of North America, 16(2), 271-283.

Klein, C. H., \& Bloch K. V. (2006). Estudos seccionais. In R. A. Midronho, K. V. Bloch, R. R. Neves \& G. L. Werneck (Eds.). Epidemiologia (p.125-150). São Paulo: Atheneu.

Kraemer, H. C., Kazdin, A. E., Offord, D., Kessler, R., Jensen, P., \& Kupfer, D. J. (1997). Coming to terms with the terms of risk. Archives of General Psychiatry, 54(4), 337-343.

Marturano, E. M., \& Loureiro, S. R. (2003). O desenvolvimento sócio-emocional e as queixas escolares. In: A. Del Prette \& Z. A. P. Del Prette (Orgs.), Habilidades sociais, desenvolvimento e aprendizagem (pp.259-291). Campinas: Alínea.

Medeiros, P. C., Loureiro, S. R., Linhares, M. B. M., \& Marturano, E. M. (2000). A auto-eficácia e os aspectos comportamentais de crianças com dificuldade de aprendizagem. Psicologia: Reflexão e Crítica, 13(3), 327-336.

Milán, S., García, E., Hurtado, J. A., Morilla, M., \& Sepúlveda, P. (2006). Victimología infantil. Cuadernos de Medicina Forense, 12(43-44), 7-19.

Molina, R. C. M., \& Del Prette, Z. A. P. (2006). Funcionalidade da relação entre habilidades sociais e dificuldades de aprendizagem. Psico-USF, 11(1), 53-63.

Murta, S. G. (2007). Programas de prevenção a problemas emocionais e comportamentais em crianças e adolescentes: lições de três décadas de pesquisa. Psicologia: Reflexão e Crítica, 20(1), 01-08.

O'Connell, M. E., Boat, T., \& Warner, K. (Eds.). (2009). Prevention mental, emotional, and behavioral disorders among young people: Progress and possibilities. Washington, D.C.: The National Academic Press.

Pacheco, J. T. B., Teixeira, M. A. P., \& Gomes, W. B. (1999). Estilos parentais e desenvolvimento de parenting styles and development of social skills during adolescence. Psicologia: Teoria e Pesquisa, 15(2), 117-126.

Paula, C. S., Duarte, C. S., \& Bordin, I. A. S. (2007). Prevalence of mental health problems in children and adolescents from the outskirts of São Paulo City and estimation of service need and capacity. Revista Brasileira de Psiquiatria, 29(1), 11-17.

Paula, C. S., Miranda, C. T., \& Bordin, I. A. S. (2010). Saúde mental na infância e adolescência: revisão dos estudos epidemiológicos brasileiros. In O. Tanaka, L. Yoshimi, \& E. Ribeiro (Orgs.), Atenção em saúde mental para crianças e adolescentes no SUS (pp.75-92). São Paulo: Editora Hucitec.

Paula, C. S., Vedovato, M. S., Bordin, I. A. S., Barros, M. G. S. M., D'Antino, M. E. F., \& Mercadante, M. T. (2008). Mental health and violence among sixth grade students from a city in the state of São Paulo. Revista de Saúde Pública, 42(3), 524-528.

Razzouk, D., Zorzetto, R., Dubugras, M. T, Gerolin, J., \& Mari, J. J. (2006). Mental health and psychiatry research in Brazil: Scientific production from 1999 to 2003. Revista de Saúde Pública, 40(Esp.), 93-100.

Rios, K. S. A., \& Williams, L. C. A. (2008). Intervenção com famílias como estratégia de prevenção de problemas de comportamento em crianças: uma revisão. Psicologia em Estudo, 13(4), 799-806.

Rocha, M. M., Pereira, R. F., Arantes, M. C., \& Silvares, E. F. M. (2010). Guia para profissionais da saúde mental sobre o Sistema Achenbach de Avaliação Empiricamente Baseada (ASEBA). São Paulo: Universidade de São Paulo.

Sá, D. G. F., Bordin, I. A. S., Martin, D., \& Paula, C. S. P. (2010). Fatores de risco para problemas de saúde mental na infância/adolescência. Psicologia: Teoria e Pesquisa, 26(4), 643-652.

Santos, E. O. L., \& Silvares, E. F. M. (2006). Crianças encaminhadas para clínicas um estudo comparativo da percepção de seus pais. Psicologia: Reflexão e Crítica, 19(2), 277-282.

Santos, P. L., \& Graminha, S. S. V. (2006). Problemas emocionais e comportamentais associados ao baixo rendimento acadêmico. Psicologia em Estudo, 11(1), 101-109.

Tramontina, S., Martins, S., Michalowski, M. B., Ketzer, C. R., Eizirik, M., Biederman, J., \& L. A. Rohde (2002). Estimated mental retardation and school dropout in a sample of students from state public schools in Porto Alegre, Brazil. Revista Brasileira de Psiquiatria, 24(4), 177-181.

Weare, K., \& Nind, M. (2012). Mental health promotion and problem prevention in schools: What does the evidence say? Health Promotion International, 26(Suppl.1), i29-i69.

Recebido em: 17/12/2012

Versão final em: 21/10/2013

Aprovado em: 13/11/2013 